\title{
Biogeographical aspects of drosophilids (Diptera, Drosophilidae) of the Brazilian savanna
}

\author{
Natália Barbi Chaves ${ }^{1} \&$ Rosana Tidon ${ }^{2}$
}

${ }^{1}$ Programa de Pós-graduação em Ecologia (PPG-ECL), Instituto de Biologia (IB), Universidade de Brasília (UnB), DF. Brasil. natalia.barbi@ pop.com.br

${ }^{2}$ Departamento de Genética e Morfologia (GEM), Instituto de Ciências Biológicas, Universidade de Brasília. Brasília-DF. Brasil. Caixa Postal 04457, 70904-970. rotidon@unb.br

\begin{abstract}
Biogeographical aspects of drosophilids (Diptera, Drosophilidae) of the Brazilian Savanna. Some species of drosophilids have been intensely studied, especially in the areas of genetics and molecular biology. However, little is known about their ecology and biogeography. Here, we revise the records of Drosophilidae species in the Cerrado domain, in order to organize the available information and to recognize patterns of geographic distribution and endemism. The 43 sampled sites in this domain reveal 100 species of drosophilids ( 10 of them exotic to the Neotropical region), distributed in eight genera. The collections were concentrated mainly in the States of Goiás (including the Federal District) and Minas Gerais. Serra do Cipó is the site with the highest richness of these insects. Of the 90 neotropical species found in the Cerrado, 77 also occur outside this domain, most of which are in the Atlantic Forest. Since extensive areas of this domain were poorly sampled for drosophilids and most studies used only baited traps to collect the flies, this absence of endemism should be carefully considered. It is recommended new inventories in poorly sampled regions, especially in the northeastern part of the domain. Also diversification of collection methods and improvement in the taxonomy of these flies are proposed. These directions should provide a significant increase in the list of species of drosophilids of the Cerrado; and thus, increase our knowledge about its biodiversity.
\end{abstract}

KEYWORDS. Drosophila; exotic species; neotropical species; pattern of distribution; Zaprionus.

RESUMO. Aspectos biogeográficos dos drosofilídeos (Diptera, Drosophilidae) da savana brasileira. Algumas espécies de drosofilídeos têm sido intensamente estudadas, principalmente nas áreas de genética e biologia molecular. Entretanto, a ecologia e biogeografia do grupo ainda são pouco conhecidas. Aqui revisamos os registros das espécies de Drosophilidae no domínio do Cerrado, visando organizar a informação disponível e reconhecer eventuais padrões de distribuição geográfica e endemismos. As 43 localidades amostradas no domínio apresentaram 100 espécies de drosofilídeos (10 delas exóticas à região Neotropical), distribuídas em oito gêneros. As coletas estão concentradas principalmente nos Estados de Goiás (incluindo Distrito Federal) e Minas Gerais. A Serra do Cipó é o local com maior riqueza desses insetos. Das 90 espécies neotropicais encontradas no Cerrado, 77 também ocorrem fora do domínio, a maioria na Mata Atlântica. Como áreas extensas do domínio foram pouco amostradas para drosofilídeos e a maior parte dos estudos utilizaram apenas iscas de banana para coletar as moscas, a ausência de endemismos deve ser analisada com precaução. São recomendados novos inventários em regiões pobremente amostradas, especialmente na parte noroeste do domínio. Também são propostas a diversificação dos métodos de coleta e o aumento do esforço de identificação taxonômico dessas moscas. Essas diretrizes devem permitir um aumento significativo na lista de espécies de drosofilídeos do Cerrado e, consequentemente, no conhecimento da biodiversidade do domínio.

PALAVRAS-CHAVE. Drosophila; espécies exóticas; espécies neotropicais; padrões de distribuição; Zaprionus.

Drosophilids are excellent biological models. These flies are small (on average 3-5mm), numerous, easily collected and manipulated, and have a short life cycle (ca. 10-20 days) which make it possible to obtain several generations in a short period of time (Powell 1997). Thus, species of the family Drosophilidae, particularly of the genus Drosophila, have been extensively used in studies of several disciplines, especially in the areas of genetics and molecular biology, summing more than 100,000 publications up to date. There is a consensus that the full understanding of evolutionary patterns and mechanisms require relating the genetics of the organism to the environment in which it is living; that is, the genetic knowledge needs to be placed in an environmental context.

In contrast to the vast amount of information known about the genetics of drosophilids, there are relatively few studies on their ecology (Val et al.1981; Powell 1997). However, in the past few years, drosophilids have increasingly turned into subject of studies that analyze niche overlapping, competition and coexistence (Sevenster \& van Alphen 1996; Toda 1999; Krijger \& Sevenster 2001). Furthermore, they also have been focus of studies on environmental bioindicators (Parsons 1995; Hoffmann et al. 2003; Mata \& Tidon 2003), as well as in studies concerning biological invasions (Tidon et al. 2003; Silva et al. 2005). As a result of the increased ecological importance of these flies in the past decade, twelve species of endemic Drosophila to Hawaii were protected by the U.S government due to its scientific value (Richardson 2006).

The study of drosophilids in the Neotropical region began in the XIX century and divided into three main periods: 1830 to 1908,1913 to 1938 , and 1941 to present. Throughout the 
first two periods, the collections were sporadic and conducted mainly by European researchers. During the third period, however, the studies were intensified and were performed by researchers from the Universidade de São Paulo (USP) and the University of Texas (USA) (Val et al. 1981, Vilela \& Bächli 1990).

The first collections in Brazil date from the second period, with important contributions by the German researcher Oswald Duda. The study of drosophilids in Brazil increased during the third period, which is divided into three phases. In the first one (1941-1959) several new species were described, and quick surveys of species were carried out in different regions of the country (Dobzhansky \& Pavan 1943; Pavan \& Cunha 1947; Pavan 1950; Frota-Pessoa 1954). This was followed by the first Brazilian studies of drosophilids' ecology (Dobzhansky \& Pavan 1950; Pavan 1959). In the second phase (1960-1980), there was a significant decrease in the bibliographical production, because many drosophilists turned to study other organisms, such as plants or human beings. In the third phase (1980-present), the study of these flies was retaken by several inventories; descriptions of species, and a deeper understanding of the ecology of these flies (Sene et al. 1980; Vilela et al. 1983; Saavedra et al. 1995; Medeiros e Klaczko 2004; Silva et al. 2005; Mateus et al. 2006; Tidon 2006). Throughout the literature, however, this information is fragmented and dispersed, and there are little studies focusing on the geographical distribution of drosophilid species.

The Brazilian Savanna, locally known as Cerrado, is the second South American domain in extension and one of 25 biodiversity hotspots in the world (Myers et al. 2000). This is the savannic formation with a high species richness, composed by a complex phytofisionomic mosaic that varies from open fields to gallery forests (Eiten 1972; Oliveira \& Marquis 2002). However, the drosophilid fauna of this region is poorly studied. The first regular collections of these insects occurred by the end of the 1990's, and they are concentrated around the Federal District (Tidon et al. 2003; Ferreira \& Tidon 2005; Tidon 2006).

Here, we compiled the available information about the geographical distribution of the drosophilids recorded in the Brazilian savanna, aiming at organizing the existent knowledge and evaluating the real distribution of the groups and the existence of eventual patterns of endemisms.

\section{METHODS}

First, a database of drosophilid species recorded in the Cerrado was organized based on the published records from 1946 to 2006 and on the collection maintained by the Laboratory of Evolutionary Biology at the Universidade de Brasília. There were no distinctions made regarding collection methods or its periodicity. When the collection sites were not georeferenced in the source of information, we used preferentially the coordinates available in the "Gazetteer Brazil Folder" software and in the "Getty Thesaurus of Geographic Names" website. References to the taxonomic authorities for drosophilid species can be found in the appendix.
The area considered in this compilation followed the morphoclimatic and phytogeographic domain proposed by Ab'Saber (1977, 2003), defined as "an area of sub-continental extent with characteristic features of topography, drainage, climate, soil and vegetation". In this study, the Cerrado (Figure 1) is considered representative of the nuclear area of the Cerrado biome; therefore, transition areas and isolated fragments were not included in the compilation. The drosophilid species recorded in the Cerrado had its occurrence investigated also in the domains of the Amazon forest (only in the Brazilian territory), Caatinga, Chaco, Atlantic forest, Araucaria forest, and Campos Sulinos (sensu Ab'Saber op. cit.).

Evaluation of the state of the knowledge. The family Drosophilidae includes 78 genera that encompass more than 3,800 species distributed worldwide, in which some are cosmopolitan and others endemic to certain areas (Bächli 2007). In Brazil, there are currently about 300 recorded drosophilid species, distributed in 17 genera. The Atlantic forest has the highest recorded drosophilid diversity of all domains, with 235 species belonging to 14 genera. In contrast, the Cerrado has a lower recorded richness of drosophilid species (100 species in 8 genera, Table I). This difference among the domains is mostly due to differences in sampling efforts. For the Atlantic forest, which covers about 650,000 $\mathrm{km}^{2}$, there are more than 170 recorded sampled sites, while for the Cerrado, which covers about 1.9 million $\mathrm{km}^{2}$, there are only 43 sampled sites registered (Table II).

The sites, where collections of drosophilids occurred, are distributed unevenly across the Cerrado area (Table II, fig. 2). There is a concentration of collection efforts in the States of Goiás and Minas Gerais, besides the Federal District, that together include 30 (70\%) of the 43 sampled sites. Among the areas with large sampling efforts is the Federal District, mainly the National Park of Brasília (PNB) and the Ecological Reserve of IBGE (RECOR-IBGE), which are regularly sampled using traps (1998 to present, see fig. 2). These areas also include increasing number of studies exploring the potential breeding sites of drosophilids. Another well studied region is the Serra do Cipó in Minas Gerais, which received expeditions in 1977, 1978, 1981 and 1989 (Tidon-Sklorz et al. 1994) and, more recently, from 1996 to 1998 (Vilela \& Mori 1999). Another reasonably well sampled region is the state of Rondônia, where researchers from the University of Brasília collected drosophilids in 2000 and 2001.

In the remaining States of the Federation, there are little sampled sites and great areas of gaps. The most critical situations are found in the States of Mato Grosso, Maranhão, Tocantins, Pará, and Piauí (Figs. 1 and 2); the last two States do not have any published record of drosophilids for the Cerrado. Besides of being poorly surveyed, two more problems are faced regarding the diversity of drosophlids in Cerrado. First, the existent records are not always results from an inventory, but can have originated from casual collections. For instance, the only collection in the municipal district of 


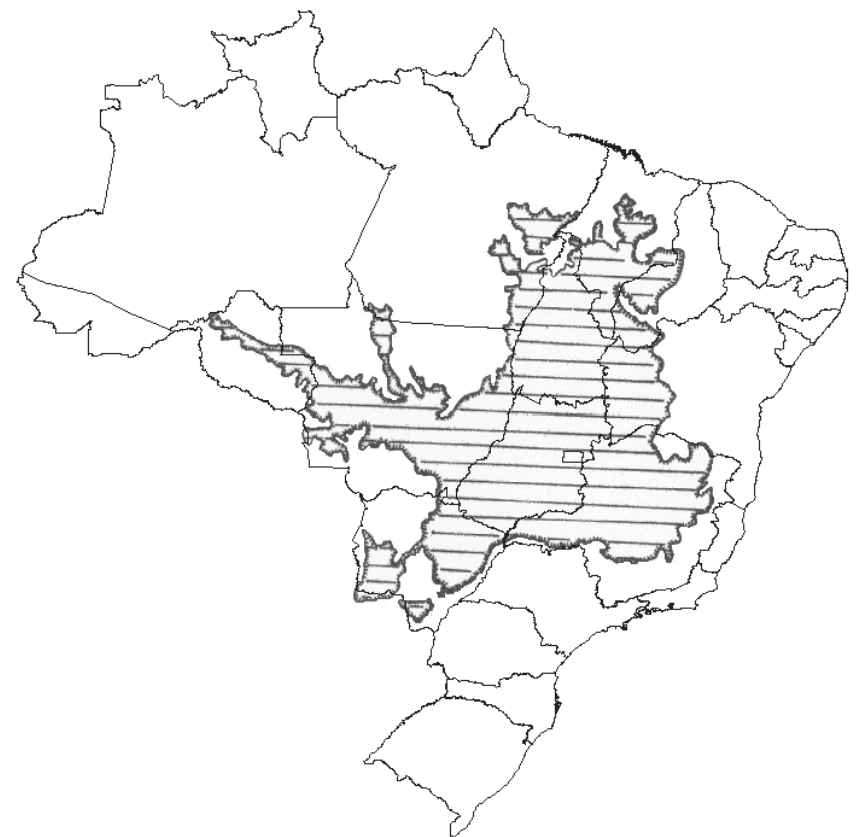

Fig. 1. Area of the domain of the Brazilian Savanna (adapted from Ab'Saber 1977).

Barra do Garças (MT) resulted from casual records of $D$. flexa, a species collected in an area of corn cultivation in the edge of a highway in 1986 (Vilela \& Bächli 2000). Another similar case is D. eleonorae, whose larvae were collected in bat guano by a speleologist group in expedition to the cities of Unaí, MG and Padre Bernardo, GO. These two records figure as the only collecting sites of drosophilids in both locations (Tosi et al. 1990). Second, there are sites sampled decades ago when the amount of described species was sensibly smaller than the current number of described drosophilids. This is the case in Carolina, MA, where a fast inventory was accomplished in the 1950's (Pavan 1959), as well as in Bela Vista, Caracol, Campo Grande, and Miranda (all in MS), and Barreira (BA), where quick inventories were carried out in the 1970's (Sene et al. 1980). These two aspects surely affect the number of species recorded at these places, because dozens of new species were ever since described and short inventories on few sites may not reflect the whole range of species diversity and distribution of a certain region.

Of the 43 sites listed in this study, only ten $(23 \%)$ were sampled for more than one month (Table II). Several of the drosophilid records were done in a one-day trip, or at random, as exemplified previously. The richness of drosophilids is strongly influenced by the regime of rains, reducing in the dry season and increasing again in the rainy season (Tidon 2006). Therefore, it is likely that the known drosophilid richness is subset of the real richness of these insects in the Cerrado.

The use of baited traps is another source promoting a subestimative of the current richness of drosophilids. Although it is widely known that traps attract species in a very selective way, few researchers collected potential breeding sites of these insects, such as decaying fruits, flowers, and mushrooms.
During a short sampling period on mushrooms, Roque et al. (2006) registered nine new occurrences of species of Drosophilidae, including rare genera, for the Cerrado. Future collections in these and other substrata should reveal the occurrences of drosophilids not yet known for the Cerrado.

Fauna of drosophilids in the Cerrado. There are 43 sites in the Cerrado with records of drosophilids (Table II, Fig. 2). Of the 100 species recorded in the domain, 90 are endemic to the neotropics and ten are exotic to this region (Table I). The exotic species are Zaprionus indianus, Scaptodrosophila latifasciaeformis and eight species of the genus Drosophila, which are distributed in three subgenera Dorsilopha (D. busckii), Drosophila (D. immigrans and D. virilis) and Sophophora (D. ananassae, D. kikkawai, D. malerkotliana, D. melanogaster, and D. simulans). The neotropical species, on the other hand, are distributed into six genera: Drosophila (predominant genus, with 74 species distributed in three subgenera), Hirtodrosophila, Leucophenga, Rhinoleucophenga, Scaptomyza and Zygothrica, given that the last five genera (except for Zygothrica, recorded in 1980) were recently recorded for the first time in this domain (Table I).

Two species of Afrotropical origin are thoroughly distributed and abundant in the Cerrado. Drosophila simulans is the first invader species of drosophilids recorded in Brazil (Pavan 1959), and it used to be the dominant exotic species in many collections (Sene et al. 1980; Tidon-Sklorz et al. 1994; Vilela \& Mori 1999). With the invasion of Zaprionus indianus towards the end of the1990's (Vilela 1999), there was a change in the drosophild assemblages, since this last species became very common in several collections of the country (Silva et al. 2005). Currently, $Z$. indianus is one of the dominant species in the Cerrado, mainly in open vegetations and during the wet season (Tidon et al. 2003).

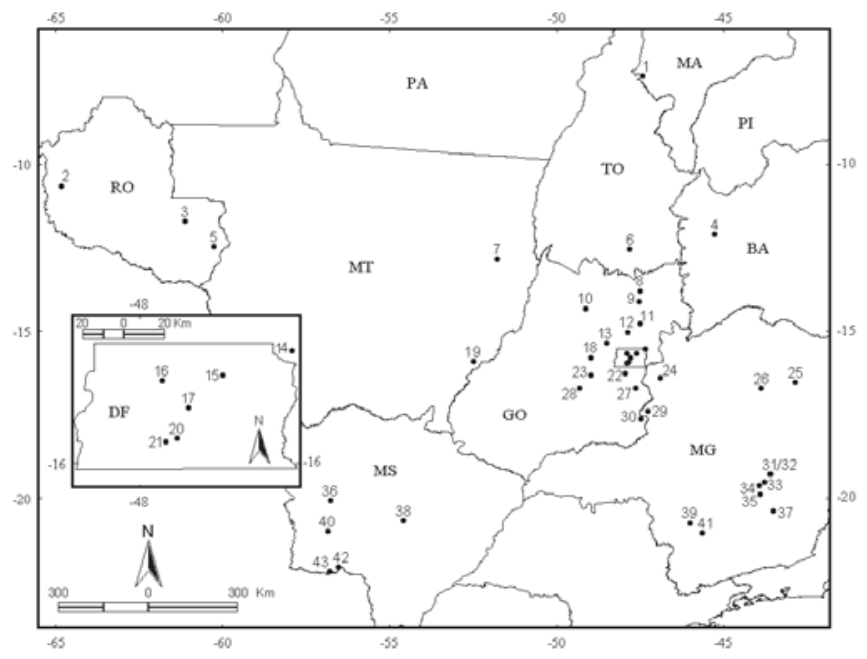

Fig. 2. Sites of the Brazilian Savanna with records of drosophilids. The codes follow Table II. In proeminence, the Federal District. 
Table I. Drosophilid species recorded in the Brazilian Savanna (with reference to the sampling site and source of information), and presence of the species in other South American domains. Exotic species are underlined. AF (Atlantic forest), AM (Brazilian Amazon), AR (Araucárias), CA (Caatinga), CH (Chaco Central), CS (Campos Sulinos). The authorships of the species are given in the appendix.

\begin{tabular}{|c|c|c|c|c|c|c|c|c|}
\hline \multicolumn{2}{|l|}{ Sites of the Cerrado } & \multirow[b]{2}{*}{ References $^{2}$} & \multicolumn{6}{|c|}{ Sites in other domains } \\
\hline Species & Sites $^{1}$ & & $\mathbf{A F}$ & $\mathbf{A M}$ & $\mathbf{A R}$ & $\mathbf{C A}$ & CH & $\mathrm{CS}$ \\
\hline D. albirostris & 31 & 22 & & & & & & \\
\hline D. ananassae & 31 & 22 & $\mathrm{x}$ & $\mathrm{x}$ & $\mathrm{x}$ & $\mathrm{x}$ & & \\
\hline D. annulimana & 31 & 24 & $\mathrm{x}$ & $\mathrm{x}$ & & & & \\
\hline D. antonietae & 2,40 & $21,26,34$ & $\mathrm{x}$ & & $\mathrm{x}$ & & $\mathrm{x}$ & $\mathrm{x}$ \\
\hline D. aragua & 21 & 33,34 & $\mathrm{x}$ & & & & $\mathrm{x}$ & \\
\hline D. arapuan & 31 & 33,34 & $\mathrm{x}$ & & & & & \\
\hline D. ararama & $16,21,31,43$ & 24 & $\mathrm{x}$ & & & $\mathrm{x}$ & $\mathrm{x}$ & \\
\hline D. arauna & 16,21 & 33,34 & $\mathrm{x}$ & & & & & \\
\hline D. atrata & $15,16,21$ & 33,34 & $\mathrm{x}$ & & & & & \\
\hline D. aureata & 31 & 24 & & & & & & \\
\hline D. austrosaltans & $15,31,43$ & $11,16,24,34$ & $\mathrm{x}$ & $\mathrm{x}$ & & & & \\
\hline D. bandeirantorum & $4,16,17,21,31$ & $16,22,33,34$ & $\mathrm{x}$ & & $\mathrm{x}$ & & $\mathrm{x}$ & \\
\hline D. bifilum & 26,31 & 8,24 & $\mathrm{x}$ & & & & & \\
\hline D. bocainensis & $12,16,21$ & $3,33,34$ & $\mathrm{x}$ & & $\mathrm{x}$ & & & \\
\hline D. bocainoides & 31 & 24 & $\mathrm{x}$ & & & & & \\
\hline D. borborema & 25 & 23 & & & & $\mathrm{x}$ & & \\
\hline D. bromelioides & 9,31 & 24,34 & $\mathrm{x}$ & & & & & \\
\hline D. busckii & $11,12,15,16,17,20,21,25,29,30,31,38$ & $10,16,22,24,26,29,31,34$ & $\mathrm{x}$ & & $\mathrm{x}$ & $\mathrm{x}$ & $\mathrm{x}$ & \\
\hline D. buzzatii & $4,25,31,32,43$ & $17,18,22,2324$ & $\mathrm{x}$ & & $\mathrm{x}$ & $\mathrm{x}$ & $\mathrm{x}$ & $\mathrm{x}$ \\
\hline D. canalinea & 12 & 3 & $\mathrm{x}$ & $\mathrm{x}$ & & $\mathrm{x}$ & & \\
\hline D. caponei & 31 & 22,24 & $\mathrm{x}$ & & & & & \\
\hline D. capricorni & 12,31 & $3,10,16,22,24$ & $\mathrm{x}$ & $\mathrm{x}$ & & $\mathrm{x}$ & & \\
\hline D. cardini & $\begin{array}{l}2,4,5,8,11,16,17,20,21,25,29,30,31 \\
34,38,42,43\end{array}$ & $22,23,24,27,29,31,32,34$ & $\mathrm{x}$ & $\mathrm{x}$ & $\mathrm{x}$ & $\mathrm{x}$ & $\mathrm{x}$ & \\
\hline D. cardinoides & $1,17,21,23,31,36,38,43$ & $10,22,24,27,31,33,34$ & $\mathrm{x}$ & $\mathrm{x}$ & $\mathrm{x}$ & $\mathrm{x}$ & $\mathrm{x}$ & \\
\hline D. coroica & 38 & 18,34 & $\mathrm{x}$ & & & & $\mathrm{x}$ & \\
\hline D. dreyfusi & 31 & 24 & $\mathrm{x}$ & & & & & \\
\hline D. eleonorae & $13,14,24$ & 20 & $\mathrm{x}$ & $\mathrm{x}$ & & $\mathrm{x}$ & & \\
\hline D. equinoxialis & 1,6 & 1 & & $\mathrm{x}$ & & & & \\
\hline D. flexa & 19 & 25 & $\mathrm{x}$ & $\mathrm{x}$ & & & $\mathrm{x}$ & \\
\hline D. fumipennis & $16,17,21,23,31$ & $3,10,16,22,34$ & $\mathrm{x}$ & $\mathrm{x}$ & $\mathrm{x}$ & & & \\
\hline D. fuscolineata & $16,21,23,29,31$ & $3,, 22,24,33,34$ & $\mathrm{x}$ & $\mathrm{x}$ & & & & \\
\hline D. gouveai & $4,27,39$ & 21,26 & $\mathrm{x}$ & & & $\mathrm{x}$ & $\mathrm{x}$ & \\
\hline D. griseolineata & 31 & $16,22,24$ & $\mathrm{x}$ & & $\mathrm{x}$ & & & \\
\hline D. guaraja & 2,31 & $22,24,34$ & $\mathrm{x}$ & & $\mathrm{x}$ & & & \\
\hline D. guaru & $11,16,21$ & 33,34 & $\mathrm{x}$ & & & & & \\
\hline D. hydei & $4,11,15,16,17,20,21,25,29,30,31,32$ & & & & & & & \\
\hline & 33,38 & $17,18,22,23,29,31,34$ & $\mathrm{x}$ & & & $\mathrm{x}$ & $\mathrm{x}$ & \\
\hline D. immigrans & $11,15,16,17,18,21,23,29,30,31,38$ & $3,16,22,24,31,34$ & $\mathrm{x}$ & & $\mathrm{x}$ & $\mathrm{x}$ & $\mathrm{x}$ & \\
\hline D. impudica & $4,42,43$ & 16 & & $\mathrm{x}$ & & $\mathrm{x}$ & & \\
\hline D. ivai & 42 & 17 & $\mathrm{x}$ & & & & & \\
\hline D. kikkawai & $17,28,35,42$ & 6,16 & $\mathrm{x}$ & & & & $\mathrm{x}$ & \\
\hline D. maculifrons & $16,17,20,21,23,29,31,38,43$ & $3,10,16,22,24,29,33,34$ & $\mathrm{x}$ & & $\mathrm{x}$ & & $\mathrm{x}$ & \\
\hline D. malerkotliana & $2,4,16,17,21,25,31,34,42,43$ & $16,22,23,24,31,34$ & $\mathrm{x}$ & $\mathrm{x}$ & $\mathrm{x}$ & $\mathrm{x}$ & $\mathrm{x}$ & \\
\hline$\overline{\text { D. medioimpressa }}$ & $21,26,31$ & $8,24,34$ & $\mathrm{x}$ & & & & & \\
\hline D. mediopicta & 31 & 24 & $\mathrm{x}$ & & $\mathrm{x}$ & & & \\
\hline D. mediopunctata & $15,16,21,31$ & $22,24,33,34$ & $\mathrm{x}$ & & $\mathrm{x}$ & $\mathrm{x}$ & & \\
\hline D. mediostriata & $6,16,17,20,21,23,26,29,31,41$ & $3,4,8,10,22,24,29,32,34$ & $\mathrm{x}$ & & $\mathrm{x}$ & $\mathrm{x}$ & $\mathrm{x}$ & \\
\hline D. melanogaster & $16,17,31,34$ & $16,22,24,31,34$ & $\mathrm{x}$ & & & $\mathrm{x}$ & $\mathrm{x}$ & \\
\hline D. mercatorum & $\begin{array}{l}2,3,4,5,8,11,15,16,17,20,21,22,25 \\
29,30,31,32,33,34,38,43\end{array}$ & $18,23,22,23,24,29,31,34$ & $\mathrm{x}$ & $\mathrm{x}$ & $\mathrm{x}$ & $\mathrm{x}$ & $\mathrm{x}$ & $\mathrm{X}$ \\
\hline D. meridionalis & $29,30,31,32$ & $18,17,22,34$ & $\mathrm{x}$ & & & $\mathrm{x}$ & $\mathrm{x}$ & $\mathrm{x}$ \\
\hline D. mesostigma & 21,31 & 22,33 & $\mathrm{x}$ & $\mathrm{x}$ & & & & \\
\hline D. nappae & 31 & 30 & $\mathrm{x}$ & & $\mathrm{x}$ & & & \\
\hline D. nebulosa & $\begin{array}{l}1,2,3,4,5,8,11,15,16,17,20,21,23 \\
25,29,31,34,38,43\end{array}$ & $\begin{array}{l}3,10,16,22,23,24,29,31 \\
32,34\end{array}$ & $\mathrm{x}$ & $\mathrm{x}$ & $\mathrm{x}$ & $\mathrm{x}$ & $\mathrm{x}$ & \\
\hline D. neocardini & 21,31 & 24,34 & $\mathrm{x}$ & $\mathrm{x}$ & & & & \\
\hline
\end{tabular}


Table I. Cont.

Sites of the Cerrado

\begin{tabular}{ll}
\hline Species & Sites $^{1}$ \\
\hline D. neocordata & $26,42,43$ \\
D. neoelliptica & 23,31 \\
D. neoguaramuru & 21 \\
D. nigricruria & $4,21,23,29,30,31,32$ \\
D. onca & 16,31 \\
D. ornatifrons & $15,16,17,20,21,31$ \\
D. pagliolii & 17 \\
D. pallidipennis & $16,17,21,23,25,31$ \\
D. parabocainensis & 26,31 \\
D. paraguayensis & $15,16,17,21,26,31$ \\
D. paramediostriata & 16 \\
D. paranaensis & $17,21,22,31,38,43$ \\
D. paulistorum & $1,4,6,21,23,41$ \\
D. polymorpha & $4,8,11,16,17,20,21,23,25,29,30,31$, \\
& $34,38,43$ \\
D. prosaltans & $1,2,3,4,6,8,11,16,17,21,29,31,34,38$, \\
& 43
\end{tabular}

D. pseudorepleta 31

D. repleta $\quad 16,21,31,38$

D. rosinae 31

D. schildi $\quad 16,17,21,23$

D. serido $\quad 12,31,32$

D. seriema 25, 31,32

D. simulans $\quad 2,3,4,5,8,11,15,16,17,20,21,25,29$, $30,31,34,38,43$

D. sturtevanti $\quad 1,2,3,4,5,8,11,15,16,17,20,21,23,25$, $29,30,31,34,38,42,43$

D. trapeza

D. tropicalis 31

D. unipunctata $1,4,6,23$

D. virilis

31

D. willistoni

31

D. zotti

$1,4,6,16,17,21,23,26,31,41$

H. morgani

31

H. pleuralis

17

L. bimaculata

17

L. maculosa

17

L. montana

17

L. ornati

L. varia 17

R. matogrossensis 36

R. nigrescens $\quad 36$

R. obesa $\quad 36$

R. personata $\quad 36$

$\underline{\text { S. latifasciaeformis }} \quad 1,2,3,4,8,11,15,16,17,20,21,23,25$,

$$
29,31,34,38,42,43
$$

S. nigripalpis 37

Z. indianus

$2,3,5,15,16,17,20,21,29,30$

Z. dispar 10

Z. microeristes 7

Z. poeyi $\quad 17$

Z. prodispar

7
17
7

References $^{2}$

9,16

$4,11,24$

34

$3,10,17,18,22,24,34$

24,33

$22,24,29,32,33,34$

13

$3,16,18,22,23,24,33,34$

$12,22,24$

$9,24,32,33,34$

33

$16,17,18,22,24,31,33,34$

15,24

$3,9,16,22,23,24,29,31,34$

$10,11,16,22,24,31,34$

22,24

$17,24,34$

24

$5,32,33,34$

$21,22,34$

$21,23,24$

$16,22,23,24,29,31,34$

$3,10,11,16,22,23,24,29$, $31,32,34$

24

1,15

24

22

$1,7,15,24,32,33,34$

24

32

32

32

32

32

32

32

1

1

1

$3,10,16,22,23,29,31,34$

14

$28,29,31,34$

19

19

32

19

\begin{tabular}{cccccc}
\hline AF & AM & AR & CA & CH & CS \\
$x$ & & $x$ & & $x$ &
\end{tabular}

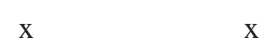

$\begin{array}{lllll}\mathrm{x} & & & & \\ \mathrm{x} & & \mathrm{x} & & \\ \mathrm{x} & & \mathrm{x} & & \\ \mathrm{x} & \mathrm{x} & \mathrm{x} & \mathrm{x} & \\ \mathrm{x} & & & & \mathrm{x} \\ \mathrm{x} & \mathrm{x} & \mathrm{x} & \mathrm{x} & \\ \mathrm{x} & & \mathrm{x} & & \\ \mathrm{x} & & \mathrm{x} & & \\ \mathrm{x} & & & & \mathrm{x} \\ \mathrm{x} & \mathrm{x} & \mathrm{x} & \mathrm{x} & \mathrm{x} \\ & & & & \end{array}$

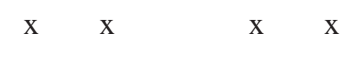

$\begin{array}{lllll}\mathrm{X} & \mathrm{X} & \mathrm{X} & \mathrm{X} & \mathrm{X}\end{array}$

$\begin{array}{lllll}x & x & x & x & \\ & & & & x\end{array}$

$\begin{array}{lllll}\mathrm{X} & \mathrm{X} & & \mathrm{X} & \\ \mathrm{X} & & & \mathrm{X} & \\ \mathrm{X} & & & & \mathrm{X} \\ \mathrm{X} & & \mathrm{X} & \mathrm{X} & \end{array}$

$x-x$

$\begin{array}{lllll}\mathrm{X} & \mathrm{X} & \mathrm{X} & \mathrm{X} & \mathrm{x} \\ \mathrm{X} & \mathrm{X} & \mathrm{X} & \mathrm{X} & \\ \mathrm{X} & & & & \\ \mathrm{X} & \mathrm{X} & & \mathrm{X} & \end{array}$

$\underline{\mathrm{x}}$

$\begin{array}{llll}\mathrm{X} & \mathrm{X} & \mathrm{X} & \mathrm{X}\end{array}$

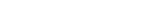

${ }^{1}$ Sites following Table II.

2. Malogolowkin (1946); 2. Burla et al. (1949); 3. Dobzhansky \& Pavan (1950); 4. Pavan (1950); 5. Burla \& Pavan (1953); 6. Freire-Maia (1953); 7. Cunha \& Dobzhansky (1954); 8. Frota-Pessoa (1954); 9. Magalhães (1956); 10. Pavan (1959); 11. Magalhães (1962); 12. Wheeler \& Magalhães (1962); 13. Cordeiro (1963); 14. Wheeler \& Takada (1966); 15. Spassky et al. (1971); 16. Sene et al. (1980); 17. Vilela (1983); 18. Vilela et al. (1983); 19. Grimaldi (1987); 20. Tosi et al. (1990); 21. Silva \& Sene (1991); 22. Tidon-Sklorz et al. (1994); 23. Tidon-Sklorz \& Sene (1995); 24. Vilela \& Mori (1999); 25. Vilela \& Bächli (2000); 26. Tidon-Sklorz \& Sene (2001); 27. Vilela et al. (2002); 28. Tidon et al. (2003); 29. Leão \& Tidon (2004); 30. Vilela et al. (2004); 31. Ferreira \& Tidon (2005); 32. Roque et al. (2006); 33. Tidon (2006); 34. collection of the Laboratory of Evolutionary Biology - UnB (1998-2006). 
Table II. List of sites of the Cerrado with records of drosophilids. Underlined sites were sampled for more than one month. The acronyms represent States of the Brazilian Federation: Bahia (BA), Distrito Federal (DF), Goiás (GO) Maranhão (MA), Mato Grosso (MT), Mato Grosso do Sul (MS), Minas Gerais (MG), Rondônia (RO) e Tocantins (TO).

\begin{tabular}{|c|c|c|}
\hline Site & Latitude & Longitude \\
\hline 1. Carolina, MA & $07^{\circ} 20^{\prime} \mathrm{S}$ & $47^{\circ} 25^{\prime} \mathrm{W}$ \\
\hline 2. Guarajá-Mirim, RO & $10^{\circ} 39^{\prime} \mathrm{S}$ & $64^{\circ} 50^{\prime} \mathrm{W}$ \\
\hline 3. Pimenta Bueno, RO & $11^{\circ} 43^{\prime} \mathrm{S}$ & $61^{\circ} 08^{\prime} \mathrm{W}$ \\
\hline 4. Barreiras, BA & $12^{\circ} 06^{\prime} \mathrm{S}$ & $45^{\circ} 15^{\prime} \mathrm{W}$ \\
\hline 5. Vilhena, RO & $12^{\circ} 29^{\prime} \mathrm{S}$ & $60^{\circ} 16^{\prime} \mathrm{W}$ \\
\hline 6. Paranã, TO & $12^{\circ} 33^{\prime} \mathrm{S}$ & $47^{\circ} 48^{\prime} \mathrm{W}$ \\
\hline 7. Mato Grosso, MT & $12^{\circ} 51^{\prime} \mathrm{S}$ & $51^{\circ} 47^{\prime} \mathrm{W}$ \\
\hline 8. Cavalcante, GO & $13^{\circ} 48^{\prime} \mathrm{S}$ & $47^{\circ} 30^{\prime} \mathrm{W}$ \\
\hline 9. São Jorge, GO & $14^{\circ} 08^{\prime} \mathrm{S}$ & $47^{\circ} 31^{\prime} \mathrm{W}$ \\
\hline 10. Campinorte, GO & $14^{\circ} 20^{\prime} \mathrm{S}$ & $49^{\circ} 08^{\prime} \mathrm{W}$ \\
\hline 11. São João da Aliança, GO & $14^{\circ} 46^{\prime} \mathrm{S}$ & $47^{\circ} 30^{\prime} \mathrm{W}$ \\
\hline 12. Água Fria de Goiás, GO & $15^{\circ} 01^{\prime} \mathrm{S}$ & $47^{\circ} 52^{\prime} \mathrm{W}$ \\
\hline 13. Padre Bernardo, GO & $15^{\circ} 21^{\prime} \mathrm{S}$ & $48^{\circ} 30^{\prime} \mathrm{W}$ \\
\hline 14. Formosa, GO & $15^{\circ} 32^{\prime} \mathrm{S}$ & $47^{\circ} 20^{\prime} \mathrm{W}$ \\
\hline 15. ESECAE, DF & $15^{\circ} 40^{\prime} \mathrm{S}$ & $47^{\circ} 37^{\prime} \mathrm{W}$ \\
\hline 16. PNB, DF & $15^{\circ} 40^{\prime} \mathrm{S}$ & $47^{\circ} 54^{\prime} \mathrm{W}$ \\
\hline 17, Brasília, DF & $15^{\circ} 47^{\prime} \mathrm{S}$ & $47^{\circ} 57^{\prime} \mathrm{W}$ \\
\hline 18. Pirenópolis, GO & $15^{\circ} 47^{\prime} \mathrm{S}$ & $48^{\circ} 58^{\prime} \mathrm{W}$ \\
\hline 19, Barra do Garças, MT & $15^{\circ} 54^{\prime} \mathrm{S}$ & $52^{\circ} 15^{\prime} \mathrm{W}$ \\
\hline 20. JBB, DF & $15^{\circ} 55^{\prime} \mathrm{S}$ & $47^{\circ} 50^{\prime} \mathrm{W}$ \\
\hline 21. RECOR, DF & $15^{\circ} 56^{\prime} \mathrm{S}$ & $47^{\circ} 53^{\prime} \mathrm{W}$ \\
\hline 22. Luziânia, GO & $16^{\circ} 16^{\prime} \mathrm{S}$ & $47^{\circ} 57^{\prime} \mathrm{W}$ \\
\hline 23. Anápolis, GO & $16^{\circ} 19^{\prime} \mathrm{S}$ & $48^{\circ} 58^{\prime} \mathrm{W}$ \\
\hline 24. Unaí, MG & $16^{\circ} 23^{\prime} \mathrm{S}$ & $46^{\circ} 53^{\prime} \mathrm{W}$ \\
\hline 25. Grão Mogol, MG & $16^{\circ} 32^{\prime} \mathrm{S}$ & $42^{\circ} 50^{\prime} \mathrm{W}$ \\
\hline 26. Montes Claros, MG & $16^{\circ} 43^{\prime} \mathrm{S}$ & $43^{\circ} 51^{\prime} \mathrm{W}$ \\
\hline 27. Cristalina, GO & $16^{\circ} 43^{\prime} \mathrm{S}$ & $47^{\circ} 37^{\prime} \mathrm{W}$ \\
\hline 28. Goiânia, GO & $16^{\circ} 43^{\prime} \mathrm{S}$ & $49^{\circ} 18^{\prime} \mathrm{W}$ \\
\hline 29. Paracatu, MG & $17^{\circ} 14^{\prime} \mathrm{S}$ & $46^{\circ} 52^{\prime} \mathrm{W}$ \\
\hline 30. Catalão, GO & $17^{\circ} 33^{\prime} \mathrm{S}$ & $47^{\circ} 38^{\prime} \mathrm{W}$ \\
\hline 31. Serra do Cipó, MG & $19^{\circ} 00^{\prime} \mathrm{S}$ & $43^{\circ} 40^{\prime} \mathrm{W}$ \\
\hline 32. Cardeal Mota, MG & $19^{\circ} 18^{\prime} \mathrm{S}$ & $43^{\circ} 35^{\prime} \mathrm{W}$ \\
\hline 33. Jaboticatubas, MG & $19^{\circ} 32^{\prime} \mathrm{S}$ & $43^{\circ} 45^{\prime} \mathrm{W}$ \\
\hline 34. Lagoa Santa, MG & $19^{\circ} 38^{\prime} \mathrm{S}$ & $43^{\circ} 55^{\prime} \mathrm{W}$ \\
\hline 35. Belo Horizonte, $\mathrm{MG}$ & $19^{\circ} 54^{\prime} \mathrm{S}$ & $43^{\circ} 54^{\prime} \mathrm{W}$ \\
\hline 36. Miranda, MS & $20^{\circ} 12^{\prime} \mathrm{S}$ & $56^{\circ} 31^{\prime} \mathrm{W}$ \\
\hline 37. Ouro Preto, MG & $20^{\circ} 23^{\prime} \mathrm{S}$ & $43^{\circ} 30^{\prime} \mathrm{W}$ \\
\hline 38. Campo Grande, MS & $20^{\circ} 24^{\prime} \mathrm{S}$ & $54^{\circ} 35^{\prime} \mathrm{W}$ \\
\hline 39. Furnas, MG & $20^{\circ} 45^{\prime} \mathrm{S}$ & $46^{\circ} 00^{\prime} \mathrm{W}$ \\
\hline 40. Serra da Bodoquena, MS & $21^{\circ} 00^{\prime} \mathrm{S}$ & $56^{\circ} 50^{\prime} \mathrm{W}$ \\
\hline 41. Boa Esperança, MG & $21^{\circ} 03^{\prime} \mathrm{S}$ & $45^{\circ} 37^{\prime} \mathrm{W}$ \\
\hline 42. Bela Vista, MS & $22^{\circ} 05^{\prime} \mathrm{S}$ & $56^{\circ} 32^{\prime} \mathrm{W}$ \\
\hline 43. Caracol, MS & $22^{\circ} 13^{\prime} \mathrm{S}$ & $56^{\circ} 47^{\prime} \mathrm{W}$ \\
\hline
\end{tabular}

D. busckii and D. immigrans are also thoughroughly distributed around the world. These species are rare in tropical climates and abundant in temperate ones, where their competitive capacities are probably higher (David \& Tsacas 1981). Their most setentrional record, in the Cerrado, is São João da Aliança (GO) (Table I). D. malerkotliana was introduced into Brasil in the 1970's, and quickly became quite abundant (David \& Tsacas op. cit.). Although this species is widely distributed and very abundant in Southern America, its frequency is very low in the Cerrado (Ferreira \& Tidon 2005; Tidon 2006). The remaining exotic species also have occurrences registered worldwide, but are quite rare in the Cerrado.

Of the 90 neotropical species listed here, 71 were recorded in a previous work that evaluated the fauna registered between 1950 and 2004 (Chaves \& Tidon 2005). The 19 species added since then are due to the sources of data used here, which included the expansion of the bibliographical revision and acquirement of new field data by the Evolutionary Biology research group of the University of Brasília. Among the neotropical drosophilids of the Cerrado there are 6 species $(0.07 \%)$ widely distributed, which have been recorded in at least 15 different sites of this domain (Drosophila cardini, $D$. mercatorum, D. nebulosa, D. polymorpha, D. prosaltans and D. sturtevanti). They are abundant in the domain and occur with high frequency in the collections.

Patterns of endemism. Most of the drosophilid species recorded in the Cerrado also occur in other morphoclimatic domains in South America, especially in the Atlantic and the Araucaria forests (Tab. I). When compared to other Brazilian domains, the Atlantic forest is definitively the region with the best collections of drosophilids in terms of size, quality, and identification efforts.

Among the investigated domains, the species that were recorded only in the Brazilian Cerrado are Drosophila albirostris, D. aureata, D. pseudorepleta, D. unipunctata (recorded only in the Serra do Cipó), and D. neocordata (found in Bela Vista and Caracol, both in the State of Mato Grosso do $\mathrm{Sul}$, and in Montes Claros, State of Minas Gerais). Among the remaining genera, Hirtodrosophila pleuralis, Leucophenga bimaculata, L. montana and L. ornativentris, were recorded only in the Federal District, whereas Rhinoleucophenga matogrossensis, $R$. nigrescens and $R$. personata are known only from its locality-type (Miranda, MS). It is possible that $D$. neocordata and the three species of Rhinoleucophenga are endemic to the Brazilian Cerrado, but more information concerning their distribution is necessary to confirm this supposition. The remaining species (D. pseudorepleta, D. unipunctata, $H$. pleuralis and L. ornativentris) also occur in other areas of the Neotropical region (Table III). The only species still not recorded for the neotropics is L. montana, formerly known only from the Neartic region.

The apparent absence of endemic drosophilid species in the Brazilian Cerrado could be an effect of poor sampling of this group: as exposed previously, of the 43 places listed here only $10(23 \%)$ were target of regular collections. Moreover, several species are not attracted to bananas, which are commonly used as baits in drosophilid traps. The study of breeding sites, also mentioned earlier, is likely to lead to the discovery of new species, as well as to diagnose endemic species in the Cerrado domain.

Despite the heterogeneous collection efforts at several sites of the Cerrado, the Serra do Cipó (MG) is conspicuous 
Table III. Sites of occurrence of drosophilid species recorded only in the Cerrado in this compilation. Source: Bächli 2007. The biogeographic regions are those from Morrone (2002).

\begin{tabular}{lll}
\hline Species & Sites & Biogeographical regions \\
\hline D. albirostris & Colombia, Costa Rica, El Salvador, Panama, Peru & Andean/Neotropical \\
D. aureata & Caribbean, Costa Rica, El Salvador, Mexico, Panama, Trinidad & Neartic/Neotropical \\
D. neocordata & Exclusive of the Cerrado & Neotropical \\
D. pseudorepleta & Paraguay & Andean/Neotropical \\
D. unipunctata & Bolivia, Colombia, Costa Rica, El Salvador, Mexico, Panama, USA, & Andean/Neotropical \\
& Venezuela & Neotropical \\
H. pleuralis & Caribbean, El Salvador & Neartic/Neotropical \\
L. bimaculata & Costa Rica, Cuba, El Salvador, Mexico & Andean/Afrotropical/Neartic/ \\
L. maculosa & Argentina, Caribbean, Chile, Cuba, Hawaii, Guyana, Mauritius, Mexico, & Neotropical/Oriental/ \\
L. montana & Peru, U.S.A. & Neartic \\
L. ornativentris & U.S.A. & Andean/Neotropical \\
R. matogrossensis & Exclivia & Neotropical \\
R. nigrescens & Exclusive of the Cerrado & Neotropical \\
R. personata & Exclusive of the Cerrado & Neotropical \\
\hline
\end{tabular}

for the high richness of drosophilid species (Tab. I). This area was sampled with banana-baited traps (Tidon-Sklorz et al. 1994; Vilela \& Mori 1999), and 61 species of the genus Drosophila were recorded. Among them, 17 species were recorded for the first time in the Brazilian Cerrado: D. albirostris, D. ananassae, $D$. annulimana, D. arapuan, D. aureata, D. bocainoides, D. caponei, D. dreyfusi, D. griseolineata, D. mediopicta, D. nappae, D. pseudorepleta, D. rosinae, D. trapeza, D. unipunctata, D. virilis, and D. zotti. In spite of the relatively small sampling efforts in this area, the Serra do Cipó presents nine more species than the Federal District, which is undoubtedly the most studied area of the Cerrado.

Silva \& Bates (2002) pointed out three areas of high richness of birds in the Brazilian Savanna: Cadeia do Espinhaço (MG), the valley of Araguaia river (MT e TO), and the valley of Paranã river (TO). It is inferable that in these valleys of the pointed rivers there is a great diversity of drosophilids, such as the Serra do Cipó. Therefore, these areas are certainly a priority for future collections of drosophilids.

Concluding remarks. The drosophilids have been unsufficiently sampled in the Cerrado. There are probably several areas with high biodiversity, such as the Serra do Cipó, which still need to be explored. It is recommended that new inventories should be carried out with priority to the states of Maranhão, Mato Grosso, Pará, Piauí and Tocantins. Furthermore, it is expect a significant increase in the list of drosophilid species in the Cerrado through the investigation of their breeding sites, progress in the identification of the flies, diversification of collection methodologies, as well the inclusion of inventories during the the dry and wet seasons.

Acknowledgements. We are grateful to the International Conservation (CI) and Conselho Nacional de Desenvolvimento Científico e Tecnológico (CNPq) for financial support, to Universidade de Brasília (UnB) for the logistics, and to Sarah Shin for the critical reading of the manuscript and English revision.

\section{REFERENCES}

Ab'Saber, A. N. 1977. Os Domínios Morfoclimáticos da América do Sul. Geomorfologia 52: 1-22.

Ab'Saber, A. N. 2003. O Domínio de Natureza no Brasil: potencialidades paisagísticas. São Paulo. Ateliê Editorial. 159p.

Bächli, G. 2007. TaxoDros: The database on taxonomy of Drosophilidae. Consulted on-line in January 2007. Available at: http://taxodros.unizh.ch/. [acessed on 08/02/2007]

Burla, H.; A. B. Cunha; A. R. Cordeiro; T. Dobzhansky; C. Malogolowkin \& C. Pavan. 1949. The willistoni group of sibling species of Drosophila. Evolution 3: 300-314.

Burla, H. \& C. Pavan. 1953. The calloptera group of species (Diptera, Drosophilidae). Revista Brasileira de Biologia 13: 291-314.

Chaves, N. B. \& R. Tidon. 2005. Drosophilidae of the Brazilian Savanna, the forgotten ecosystem. Drosophila Information Service 88: $25-27$.

Cordeiro, A. R. 1963. "Drosophila pagliolit" a new species showing unusual chromatographic pattern of phluorescent substances. Revista Brasileira de Biologia 23: 401-407.

Cunha, A. B. \& T. Dobzhansky. 1954. A further study of chromossomal polymorphism in Drosophila willistoni in its relation to the environment. Evolution 8: 119-134.

David, J. \& L. Tsacas. 1981. Cosmopolitan, subcosmopolitan and widespread species: different strategies within the drosophilid family (Diptera). Comptes rendus des seances de la Societe de Biogeographie 57: 11-26.

Dobzhansky, T. \& C. Pavan. 1943. Studies on Brazilian species of Drosophila. Boletim da Faculdade de Filosofia, Ciencias e Letras, Universidade de São Paulo 36: 7-72.

Dobzhansky, T. \& C. Pavan. 1950. Local and seasonal variations in relatives frequencies of species of Drosophila in Brazil. Journal of Animal Ecology 19: 1-14.

Eiten, G. 1972. The Cerrado vegetation of Brazil. Botanical Review 38: 201-341.

Ferreira, L. B. \& R. Tidon. 2005. Colonizing potential of Drosophilidae (Insecta, Diptera) in environments with different grades of urbanization. Biodiversity and Conservation 14: 1809-1821.

Freire-Maia, N. 1953. Frequencies of the two color forms of the Brazilian D. montium in natural populations. Drosophila Information Service 27: 90-91.

Frota-Pessoa, O. 1954. Revision of the tripunctata group of Drosophila with description of fifteen new species (Drosophilidae, Diptera). Arquivos do Museu Paranaense 10: 253-304.

Grimaldi, D. A. 1987. Phylogenetics and taxonomy of Zygothrica 
(Diptera: Drosophilidae). Bulletin of the American Museum of Natural History 186(2): 103-268.

Hoffmann, A. A.; R. J. Hallas; J. A. Dean \& M. Schiffer. 2003. Low Potential for Climatic Stress Adaptation in a Rainforest Drosophila species. Science 301: 100-102.

Krijger, C. L. \& J. G. Sevenster. 2001. Higher species diversity explained by stronger spatial aggregation across six neotropical Drosophila communities. Ecology Letters 4: 106-115.

Leão, B. F. D. \& R. Tidon. 2004. Newly invading species exploiting native host-plants: the case of the African Zaprionus indianus (Gupta) in the Brazilian Cerrado (Diptera, Drosophilidae). Annales de la Societe Entomologique de France 40: 285-290.

Magalhães, L. E. 1956. Description of four new species of the saltans group of Drosophila (Diptera). Revista Brasileira de Biologia 16: $273-280$.

Magalhães, L. E. 1962. Notes on the taxonomy, morphology and distribution of the saltans group or Drosophila with description of four new species. University of Texas Publications 6205: 135154.

Malogolowkin, C. 1946. Sobre o gênero "Rhinoleucophenga" com descrição de cinco espécies novas (Drosophilidae, Diptera). Revista Brasileira de Biologia 6: 415-426.

Mata, R. A. \& R. Tidon. 2003. Insetos Informantes. Ciência Hoje 32: $64-65$.

Mateus, R. P.; M. L. T. Buschini \& F. M. Sene. 2006. The Drosophila community in xerophytic vegetations of the upper ParanaParaguay River Basin. Brazilian Journal of Biology 66: 719 729.

Medeiros, H. F. \& L. B. Klaczko. 2004. How many species of Drosophila (Diptera, Drosophilidae) remain to be described in the forests of São Paulo, Brazil? Species list of three forest remnants. Biota Neotropica 4: 1-12.

Morrone, J. J. 2002. Biogeographical regions under track and cladistic scrutiny. Journal of Biogeography 29: 149-152.

Myers, N.; R. A. Mittermeyer; C. G. Mittermeyer; G. A. B. Fonseca \& J. Kent. 2000. Biodiversity spots for conservation priorities. Nature 403: 853-858.

Oliveira, P. S. \& R. J. Marquis. 2002. The Cerrados of Brazil. Ecology and Natural History of a Neotropical Savanna. New York. Columbia University Press. 424 p.

Parsons, P. A. 1995. Evolutionary response to drought stress conservation implications. Biological Conservation 74: $21-$ 27.

Pavan, C. 1950. Espécies brasileiras de Drosophila II. Boletim da Faculdade de Filosofia, Ciências e Letras da Universidade de São Paulo 111: 3-36.

Pavan, C. 1959. Relações entre populações naturais de Drosophila e o meio ambiente. Boletim da Faculdade de Filosofia, Ciências e Letras da Universidade de São Paulo 221: 1-81.

Pavan, C. \& A. B. Cunha. 1947. Espécies brasileiras de Drosophila. Boletim da Faculdade de Filosofia, Ciencias e Letras, Universidade de São Paulo 86: 3-46.

Powell, J. R. 1997. Progress and Prospects in Evolutionary Biology: the Drosophila Model. New York. Oxford University Press. xiv +562 p.

Richardson, M. 2006. Endangered and Threatened Wildlife and Plants; Determination of Status for 12 Species of Picture-Wing Flies From the Hawaiian Islands. Federal Register 71: 26835-26852.

Roque, F.; R. Figueiredo \& R. Tidon. 2006. Nine new records of drosophilids in the Brazilian savanna. Drosophila Information Service 89: $1-3$.

Saavedra, C. C. R.; S. M. Callegari-Jacques; M. Napp \& V. L. S. Valente. 1995. A descriptive and analytical study of four Neotropical drosophilid communities. Journal of Zoological Systematics and Evolutionary Research 33: 62-74.

Sene, F. M.; F. C. Val; C. R. Vilela \& M. A. Q. R. Pereira. 1980. Preliminary data on the geographical distribution of Drosophila species whitin morpho-climatic domains of Brazil. Papéis Avulsos de Zoologia 33: 315-326.

Sevenster, J. G. \& J. J. M.van Alphen. 1996. Aggregation and coexistence.
II. A neotropical Drosophila community. Journal of Animal Ecology 65: 308-324.

Silva, A. F. G. \& F. M. Sene. 1991. Morphological geographic variability in Drosophila serido (Diptera, Drosophilidae). Revista Brasileira de Entomologia 35: 455-468.

Silva, J. M. C. \& J. M. Bates. 2002. Biogeographic Patterns and Conservation in the South American Cerrado: A tropical Savanna Hotspot. BioScience 52: 225-233.

Silva, N. M.; C. C. Fantinel; V. L. S. Valente \& V. H. Valiati. 2005. Population Dynamics of the Invasive Species, Zaprionus indianus (Gupta) (Diptera: Drosophilidae) in Communities of Drosophilids on Porto Alegre City, Southern of Brazil. Neotropical Entomology 34: $363-374$.

Spassky, B.; R. C. Richmond; S. Perez-Salas; O. Pavlovsky; C. A. Mourão; A. S. Hunter; H. Hoenigsberg; T. Dobzhansky \& B. J. Ayala. 1971. Geography of sibling species related to Drosophila willistoni, and of semispecies of Drosophila paulistorum complex. Evolution 25: $129-143$.

Tidon, R. 2006. Relationships between drosophilids (Diptera, Drosophilidae) and the environment in two contrasting tropical vegetations. Biological Journal of the Linnean Society 87: 233-247.

Tidon, R.; D. F. Leite \& B. F. D. Leão. 2003. Impact of the colonisation of Zaprionus (Diptera, Drosophilidae) in different ecosystems of the Neotropical Region: 2 years after the invasion. Biological Conservation 112: 299-305.

Tidon-Sklorz, R. \& F. M. Sene. 1995. Fauna of Drosophila (Diptera, Drosophilidae) in the northern area of the "Cadeia do Espinhaco", states of Minas Gerais and Bahia, Brazil: biogeographical and ecological aspects. Iheringia, Série Zoológica 78: 85-94.

Tidon-Sklorz, R. \& F. M. Sene. 2001. Two new species of the Drosophila serido sibling set (Diptera, Drosophilidae). Iheringia, Série Zoológica 90: 141-146.

Tidon-Sklorz, R.; C. R. Vilela; F. M. Sene \& M. A. Q. R. Pereira. 1994. The genus Drosophila (Diptera, Drosophilidae) in the Serra do Cipó, State of Minas Gerais, Brazil. Revista Brasileira de Entomologia 38: 627-637.

Toda, M. J. 1999. Coexistence mechanisms of mycophagous drosophilids on multispecies fungal hosts: aggregation and resource partitioning. Journal of Animal Ecology 68: 794-803.

Tosi, D.; M. Martins; C. R. Vilela \& M. A. Q. R. Pereira. 1990. On a new cave-dwelling species of bat-guano-breeding Drosophila closely related to Drosophila repleta Wollaston (Diptera, Drosophilidae). Revista Brasileira de Genética 13: 19-31.

Val, F. C.; C. R. Vilela \& M. D. Marques. 1981. Drosophilidae of the Neotropical Region, p. 123-168. In: M. Ashburner; H. L. Carson \& J. N. Thompson (eds). The Genetics and Biology of Drosophila. London. Academic Press. vol. 3a. xvi+429 p.

Vilela, C. R. 1983. A revision of the Drosophila repleta species group. (Diptera, Drosophilidae). Revista Brasileira de Entomologia 27: $1-114$.

Vilela, C. R. 1999. Is Zaprionus indianus Gupta, 1970 (Diptera, Drosophilidae) currently colonizing the Neotropical region? Drosophila Information Service 82: 37-38.

Vilela, C. R. \& G. Bächli. 1990. Taxonomic studies on Neotropical species of seven genera of Drosophilidae (Diptera). Mitteilungen der Schweizerische Entomologischen Gesellschaft 63: 1332.

Vilela, C. R. \& G. Bächli. 2000. Morphological and ecological notes on the two species of Drosophila belonging to the subgenus Siphlodora Patterson \& Mainland, 1944 (Diptera, Drosophilidae). Mitteilungen der Schweizerische Entomologischen Gesellschaft 73: 23-47.

Vilela, C. R. \& L. Mori. 1999. The genus Drosophila (Diptera, Drosophilidae) in the Serra do Cipó: further notes. Revista Brasileira de Entomologia 43: 319-328.

Vilela, C. R.; A. F. G. Silva \& F. M. Sene. 2002. Preliminary data on geographical distribution of Drosophila species within morphoclimatic domains of Brazil III: The cardini group. Revista Brasileira de Entomologia 46: 139-148. 
Vilela, C. R.; M. A. Q. R. Pereira \& F. M. Sene. 1983. Preliminary data on geographical distribution of Drosophila species within morphoclimatic domains of Brazil II: The repleta group. Ciência e Cultura 35: 66-70.

Vilela, C. R.; V. L. S Valente \& L. Basso-da-Silva. 2004. Drosophila angustibucca Duda sensu Frota-Pessoa is na undescribed species (Diptera Drosophilidae). Revista Brasileira de Entomologia 48: $233-238$.
Wheeler, M. R. \& L. E. Magalhães. 1962. The algitans-bocainensis complex of the willistoni group of Drosophila. University of Texas Publications 6205: 155-171.

Wheeler, M. R. \& H. Takada. 1966. The nearctic and neotropical species of Scaptomyza Hardy (Diptera; Drosophilidae). University of Texas Publications 6615: 37-78.

\section{APPENDIX}

List of species of the family Drosophilidae (Diptera) cited in this article.

Drosophila albirostris Sturtevant 1921

D. ananassae Doleschall 1858

D. annulimana Duda 1927

D. antonietae Tidon-Sklorz \& Sene 2001

D. aragua Vilela \& Pereira 1982

D. ararama Pavan \& Cunha 1947

D. arauna Pavan \& Nacrur 1950

D. arapuan Cunha \& Pavan 1947

D. atrata Burla \& Pavan 1953

D. aureata Wheeler 1957

D. austrosaltans Spassky 1957

D. bandeirantorum Dobzhansky \& Pavan 1943

D. bifilum Frota-Pessoa 1954

D. bocainensis Pavan \& Cunha 1947

D. bocainoides Carson 1954

D. borborema Vilela \& Sene 1977

D. bromelioides Pavan \& Cunha 1947

D. busckii Coquillet 1901

D. buzzatii Patterson \& Wheeler 1942

D. canalinea Patterson \& Mainland 1944

D. caponei Pavan \& Cunha 1947

D. capricorni Dobzhansky \& Pavan 1943

D. cardini Sturtevant 1916

D. cardinoides Dobzanhsky \& Pavan 1943

D. coroica Wasserman 1962

D. dreyfusi Dobzanhsky \& Pavan 1943

D. eleonorae Tosi Martins Vilela \& Pereira 1990

D. equinoxialis Dobzhansky 1946

D. flexa Loew 1866

D. fumipennis Duda 1925

D. fuscolineata Duda 1925

D. gouveai Tidon-Sklorz \& Sene 2001

D. griseolineata Duda 1927

D. guaraja King 1947

D. guaru Dobzanhsky \& Pavan 1943

D. hydei Sturtevant 1921

D. immigrans Sturtevant 1921

D. impudica Duda 1927

D. ivai Vilela 1983

D. kikkawai Burla 1954

D. maculifrons Duda 1927

D. malerkotliana Parshad \& Paika 1964

D. medioimpressa Frota-Pessoa 1954

D. mediopicta Frota-Pessoa 1954

D. mediopunctata Dobzhansky \& Pavan 1943

D. mediostriata Duda 1925

D. melanogaster Meigen 1830

D. mercatorum Patterson \& Wheeler 1942

D. meridionalis Wasserman 1962

D. mesostigma Frota-Pessoa 1954
D. nappae Vilela Valente \& Basso-da-Silva 2004

D. nebulosa Sturtevant 1916

D. neocardini Streisinger 1946

D. neocordata Magalhães 1956

D. neoelliptica Pavan \& Magalhães 1950

D. neoguaramuru Frydenberg 1956

D. nigricruria Patterson \& Mainland 1943

D. onca Dobzanhsky \& Pavan 1943

D. ornatifrons Duda 1927

D. pagliolli Cordeiro 1963

D. pallidipennis Dobzanhsky \& Pavan 1943

D. parabocainensis Carson 1954

D. paraguayensis Duda 1927

D. paramediostriata Townsend \& Wheeler 1955

D. paranaensis Barros 1950

D. paulistorum Dobzhansky \& Pavan 1949

D. polymorpha Dobzanhsky \& Pavan 1943

D. prosaltans Duda 1927

D. pseudorepleta Vilela \& Bächli 1990

D. repleta Wollaston 1858

D. rosinae Vilela 1983

D. schildi Malloch 1924

D. serido Vilela \& Sene 1977

D. seriema Tidon-Sklorz \& Sene 1995

D. simulans Sturtevant 1919

D. sturtevanti Duda 1927

D. trapeza Heed \& Wheeler 1957

D. tropicalis Burla \& Cunha 1949

D. unipunctata Patterson \& Mainland 1943

D. virilis Sturtevant 1916

D. willistoni Sturtevant 1916

D. zotti Vilela 1983

Hirtodrosophila morgani (Mourão Gallo \& Bicudo 1967)

H. pleuralis (Williston 1896)

Leucophenga bimaculata (Loew 1866)

L. maculosa (Coquillett 1895)

L. montana Wheeler 1952

L. ornativentris Kahl 1917

L. varia (Walker 1849)

Rhinoleucophenga matogrossensis Malogolowkin 1946

R. nigrescens Malogolowkin 1946

R. obesa (Loew 1872)

R. personata Malagolowkin 1946

Scaptodrosophila latifasciaeformis (Duda 1940)

Scaptomyza nigripalpis Malloch 1924

Zaprionus indianus Gupta 1970

Zygothrica dispar (Wiedemann 1830)

Z. microeristes Grimaldi 1987

Z. poeyi (Sturtevant 1921)

Z. prodispar Duda 1925

Received 03/10/2007; accepted 03/06/2008 